\title{
Affordances and Constraints to E-Learning: Role of Architecture in 3D Learning Space Digital Design
}

\author{
Noha Saleeb, Georgios Dafoulas \\ Middlesex University, London, UK
}

\begin{abstract}
While abundant research has been devoted to investigate successful learning prospects of $3 D$ Virtual Learning Environments (VLES), sparse research explores users' satisfaction from buildings used within these 3DVLEs. No research depicts users' contentment levels, preferences or requirements from $3 D$ educational buildings' different constructional and architectural design elements. This paper is part of an ongoing research investigating the presence of such impact of architectural features of $3 D$ virtual educational classrooms on users' comfort within them, by recording and categorizing higher education students' space design propositions to enhance virtual campuses, internally and externally. This has potential to boost e-learning experiences within 3DVLEs analogous to the positive effect of physical real-life architecture on students' learning within their respective classrooms. The second part of this paper offers an example. For, while former research also reveals that architectural colour-design of physical learning spaces directly affects students' learning, there is no equivalent study supporting similar impact of $3 D$ virtual learning spaces' colourdesign on students in $3 D$ VLEs, whether as affordances or constraints to the e-learning process. To fill this gap, this paper examines the various positive and negative emotions expressed by students during exposure to different colours inside $3 D$ virtual educational buildings; in an attempt to determine best practices in digital colour-design of learning spaces inside $3 D$ virtual worlds to enhance students' overall e-learning experiences and satisfaction within them.
\end{abstract}

\section{Introduction}

These 3D virtual worlds, utilized as 3D Virtual Learning Environments (VLEs), constitute a newly emergent technology that has been increasingly used by hundreds of universities and pedagogical institutions over the past decade as a supplementary didactic medium for various online and hybrid courses [1]. This has necessitated erection of virtual campuses inside 3D VLEs, e.g. Second Life, where students can receive their e-learning sessions.
Creating 3D virtual campuses for real-life institutions in virtual worlds, like Second Life, has allowed designers to fantasize about endless possibilities for creating buildings not possible to erect physically. 3D VLEs have enabled the emergence of many innovative ideas in the construction and architecture of educational buildings owned and used by numerous universities based inside these worlds and from which they deliver legitimate education. Furthermore, there is a substantial difference between building within virtual worlds and in the physical world, for there are no boundaries to construction. In 3D VLEs there are no real-life constraints of budgets for buying constructional materials, no constructional permits, soil tests, engineering natural forces, material limitations, infrastructure requirements, sound, ventilation regulations or even gravity which means buildings can even be erected in mid-air. Thus a simple 3D procedure could renovate and enliven the textures of dull walls, enrich dreary window styles, open up the roof like a convertible automobile... or better still teleport the whole 3D class suddenly to find one leisurely learning from inside a pool of water surrounded by palm trees, or completely submerged underwater.

However there are currently no architectural specifications or guidelines for designing these 3D virtual buildings. They are either being created according to "real life" specifications or designers' and educators' viewpoints [2] without consideration for students' preferences and needs from 3D virtual learning spaces which, lacking in research, might be different from students' needs from physical learning spaces.

Therefore, whilst literature shows a direct effect for physical architecture on learning [3], there is no research demonstrating the effect of architecture of 3D educational buildings on users or their e-learning, or their specific opinion of the design aspects of virtual buildings generally, and educational virtual facilities specifically. With all these possibilities and lack in research, arises the need to investigate users' views and requirements from 3D virtual architectural design of buildings to issue recommendations for their future enhancement.

This study is part of an ongoing research investigating the effects of architectural design elements of 3D virtual educational facilities on the learning experience and satisfaction of students, 
hence evaluating suitability of these spaces for the elearning process, to derive best architectural digital design recommendations apt for enhancing the learning experience [4]. A design element under focus in this paper is colour of the learning space. The interior colours of "real life" physical learning spaces have been evidenced to have an impact on students' engagement, participation, understanding etc., and also evidenced to invoke various psychological reactions and emotions associated with different colours, as demonstrated subsequently [5]; for colour in design can augment and detail other visual elements of space. Complementary with former statements, there is also no corresponding research depicting effect of 3D virtual learning space colours on students [6], which is a second objective of the study at hand through exploring the positive and negative emotion-associations of students with different space colours.

From an academic perspective the creation of a 3D VLE provides an opportunity to shift the learning process into an environment that is familiar to a generation spending a significant amount of time, even daily, using 3D gaming platforms. It should be noted that several misconceptions may affect an educator's judgment when it comes to the identification of needs, skills and typical behaviour of learners in 3D worlds. These misconceptions will be discussed later in this paper.

The contribution offered by this research paper lies at the intersection of e-learning, architecture and 3D computer science virtual product digital design, focusing on closing this gap in research by extracting student preferred design factors for educational spaces and buildings.

\section{Background}

All printed material, As explicated earlier, very little research exists that investigates the effect of architectural elements (e.g. dimensions, shape, colours, textures, lighting etc.) of 3D buildings in general on users in 3D VLEs, and their satisfaction and contentment from them. Some remotely related studies explore a collaborative learning approach to digital architectural design within a 3D real-time virtual environment [7]. Others discuss systems for augmenting real-time 3D virtual environments to support the formation and compositions of architectural designs [8]. Moreover, existing tutorials illustrate how to use building tools to construct and edit within 3D VLEs, but do not offer any guidelines as to the specifications to take into consideration to make them functional, usable and acceptable by users. An individual market research, within Second Life, depicting users' reactions to preferences between realistic buildings and imaginative style buildings, only shows that users prefer realistic style buildings with a percentage of $60 \%$ more than imaginative style 3D buildings [9].

There are also only some general recommendations or guidelines offered by previous researchers interested in design of virtual environments, based on observation and interviews with 3D VLE general users (not on interaction of the learners with the environment during the e-learning process), to aid design 3D virtual educational spaces. For example Dickey [10] suggests using architectural and environmental elements such as landmarks, signs, paths and boundaries to aid students' way finding. Bridges \& Charitos [11] note that real world elements, e.g. doors, roofs, columns, structural or ornamental details, should only be used if there is a functional use for them (e.g. no door if the walls are penetrable). Minocha \& Reeves [12] further propose using "open spaces as much as possible" to accommodate flying, wide corridors, realism in design, and arrangement of spaces to follow activities performed in them. As for the factors affecting the level of engagement experienced by the learners, only pedagogical factors were identified not architectural factors.

On a more detailed level, since brain-based learning occurs via the senses, what students see, hear, feel and smell has a significant impact on their learning. Colour is thus one of the vital elements in a stimulus-rich learning environment [13]. An exploratory experiment was conducted with ninety eight college students capturing their emotional reactions to a "physical" learning environment with different colour hues: five principle hues (red, yellow, green, blue, purple), five intermediate hues (yellow-red, green-yellow, blue-green, purple-blue, and red-purple), and three achromatic neutral colours (white, gray, and black). Results conveyed that principle hues attracted highest number of positive emotional responses, followed the intermediate hues and achromatic colours. Green induced mainly positive emotions such as relaxation and comfort because of its association with nature. Green-yellow received lowest number of positive responses drawing feelings of sickness and disgust. Regarding neutral colours, white collected a large number of positive responses giving peacefulness but sometimes boredom. Purple was not favoured. Gray was associated with negative emotions including feelings of sadness, depression, boredom, and tiredness [14].

Furthermore, psychology of colour usage for websites revealed the following sentiments in connection with different colours: i) Warm colours: red - excitement and activity, pink - creativity, confidence, orange - distress. ii) Cool colours: green - peacefulness and harmony, blue - quiet, calmness, purple - beauty, inspiration. iii) Yellow-greens - least preferred, yellow can increase concentration and cheerfulness, but can be irritating. iv) Neutral: White 
- purity, Black - elegance, gloom [15]. Supplementary findings demonstrated that red causes emotional arousal, changes in heart rate, and electrical brain activity, being considered an impressive but sometimes a disturbing colour. Experiments depicting emotional associations of school students with nine different colours confirmed positive reactions for bright colours (e.g., pink, blue, red) and negative reactions for dark colours (e.g., brown, black, gray) [16]. This confirmed established results by Failey et al. [17] unveiling an association between colour choices of learning spaces and student achievement. Optical stimulation for example was evidenced to be caused by use of warm colours, and intensity of lighting invoked muscular tensions and brain activity. Cool colours and dim lighting caused reverse effects e.g. muscle relaxation and drowsiness.

Regarding the effect of age on colour preferences in the physical world, Birren [18] noted that with maturity, cooler (short wave length) hues e.g. blue, green, purple become more preferable than warmer (longer wave length) hues e.g. red orange, yellow. As for gender difference, both males and females showed highest affinity for blue followed by green hues [19].

All previous existing and absent evidence from research to the importance of $3 \mathrm{D}$ virtual space architectural elements of design on the e-learning experience, with particular attention to colour in this paper, were taken into consideration during design of this research rationale as demonstrated subsequently.

\section{Research rationale}

A qualitative research approach comprising preliminary surveys, focus groups and interviews was identified as suitable to initially discover students' preferences regarding presence of different constructional and architectural elements within 16 selectively chosen 3D university virtual campuses within Second Life. Partaking in this survey were 84 participants from the school of Engineering in Middlesex University, UK. These are divided into the following categories which correspond to the different clusters of users utilising 3D virtual university campuses to participate in online elearning sessions, for both learning and teaching.

The participants comprised 31 under graduate students, 33 post graduate students, and 20 adult learners from different age groups (30 to 60 years old). The selected 3D virtual campuses were nominated since they represent a variety of building design specifications, which were analysed in depth using the survey likert-scale questions. As a component of the administered survey questionnaire, which was issued to the participants while interactively showing them the chosen 16 3D virtual university campuses, 6 open ended questions were included to capture student and faculty preferences for educational space design, from each of the 16 3D campuses shown to them. The aim of the project was explained to students from several classes, prior to conducting the surveys, and only those volunteering to contribute remained in the survey sessions, producing the participant numbers mentioned above.

While the likert-scale questions of the survey and their results are not the focus of this current paper, the results of the open-ended questions, exemplifying students' educational space design preferences, are displayed henceforth. The questions were:

- What interior design aspects did you like most in this learning space?

- How do they make you feel (optional)?

- What interior design aspects did you dislike in this learning space?

- How do they make you feel (optional)?

- What exterior design aspects did you like most in this learning space?

- How do they make you feel (optional)?

- What exterior design aspects did you dislike in this learning space?

- How do they make you feel (optional)?

- What interior design features would you recommend for this learning space?

- What exterior design features would you recommend for this learning space?

The open ended questions were used to allow students to think freely with no inhibitions on their desires, thus opening up points for discussion that we as researchers might have overlooked and not specifically asked about within the closed options likert-scale questions.

After collection and analysis of the preliminary data from the open ended answers, 2 focus groups were arranged with 8 members from each of our 2 under graduate and post graduate groups of previous participants, and 5 individual interviews were arranged with our adult learners, also contributing previously. These numbers comprised quarter of the whole survey sample, allowing us to discuss in more detail, the users' perceptions of appropriate architectural design elements for learning spaces, which they proposed earlier.

The different approaches followed in data collection between student sampling and interviews, allowed the authors a more comprehensive understanding of how the different groups would perceive the environment differently. Although student participants were initially approached in groups who would experience the 16 environments at the same time, the research team ensured that each individual was engaged in one-to-one brief discussions while answering the open ended questions. The objective of this technique was to ensure that the purpose of each question was clear 
and to establish that the interpretation of the participant responses was accurate.

It became evident that a key difference between under graduate, post graduate students and adult learner approaches to the question was due to their different agendas while engaging with the environments. Younger students groups approached the exercise keen to share their ideas of what a learning space should look and feel like. It was obvious that their drive was to share their views for design principles that should be followed during the creation of their own space in the future. On the other hand older groups were motivated to reflect on how what was shown could affect the delivery of certain learning activities or support academic related and administrative tasks essential for them.

For the second half of this paper presenting findings on students' positive and negative associations with colour in particular(as an example of an architectural digital design element in 3D virtual learning spaces), and thus its affordances and constraints for e-learning, the following research rationale was followed.

As previously stated, the aim of this study was to investigate students' emotional associations with a diverse range of colours used in their e-learning spaces to derive best practices for 3D virtual educational space colour design for enhancement of students' learning experiences. Hence a series of controlled identical experiments were conducted using several student groups. Participants included 35 under graduates (2 groups) and 27 post graduates (2 groups) from the School of Engineering and Information Sciences at Middlesex University. Twelve colours were chosen for testing representing primary hues: red, blue, yellow; secondary hues: green, purple, orange; neutral colour: white; and light and dark hues (with white or black added to primary or secondary colours): light blue, light green, pink, grey, brown. These colours correspond to the main components of the Munsell Colour System [20] and combine warm (red, orange, yellow, brown), cool (blues, greens, purple) and neutral colors (grey and white).

In preparation for an experiment, participants went through a series of stages familiarizing with the $3 \mathrm{D}$ virtual environment and acquiring basic skills. The aim was to make the learning environment a comfortable setting for the students to ensure that perception of architectural elements was not affected by lack of experience or novice users. The experiment entailed delivering a complete e-learning session to consenting participants within a predefined learning space inside Second Life as a representative of 3D VLEs. The experiment session did not alter the learning outcomes of the course students participated in and focused only on the transition of learning tasks in the buildings in Second Life. During a session, colours of walls, floor and ceiling were changed (all the same colour) at regular intervals to immerse the students in 12 different colour environments. At the end of the session, all participants were asked to express the emotions they experienced during subjection to each of the 12 tested colours. Students were given the following list of positive versus opposing negative emotions to choose from: comfortable-irritated, attentive-sleepy, lively-tired, and interested-bored. These emotions were selected as category summarization of a list of comprehensive emotions identified by Kaya [14].

The following measures were taken into consideration to counteract for extraneous variables that could influence the emerging results. This was crucial to ensure that colour was the only independent variable affecting students' differing emotion associations with the $3 \mathrm{D}$ virtual learning space:

- The order of presenting the different colours was randomized for each group of students to prevent memory associations with order.

- All experiments were conducted using "midday" mode inside Second Life to unify amount of light and direction of shade falling on coloured walls, floor and ceiling through artificial windows thus giving the same shades of colour for all participants and replicating as close as possible the time of usual morning e-learning sessions.

- The pilots started after ensuring that all students had a harmonic experience of their displayed environment and consistency in brightness and contrast monitor settings.

- While the paper at hand focuses only on overall average results of the whole participating student body, gender of each participant was recorded as well as age group (under graduate or post graduate) to be taken into account during future data analysis.

- The cultural background of students was diversified.

- None of the participants had defective colour vision as verified with the Ishihara Colour Deficiency test taken online prior to experimenting.

- The original background material of walls, floor and ceiling was set to "none" prior to administering colour changes on them to eliminate effects of other material colours or textures on the resulting hues, thus obtaining pure shades of 3D virtual colour. Also no mixtures of colours were used between walls, floor and ceiling to eliminate mixed effects of colours on participants.

- The dimensions and shape of the e-learning space was unified for all experiments to eliminate the effect of space size factor on the results. A medium seminar sized room was chosen with rectangular shape.

- During experiment, students were asked to view the space from different angles using their camera 
controls to get a comprehensive perspective of the colours used.

- No combinations of colours were used to test impact of individual colours.

After tabulating the number of positive and negative emotion-associated votes given for each colour, the following equations were calculated, and diagrams illustrating different findings were created accordingly as demonstrated subsequently:

Percentage of importance of each colour-associated emotion:

$$
\begin{aligned}
& \text { ( No. of votes per emotion for all colours } \\
& \text { / total number of votes for all emotions for all } \\
& \text { colours) } * 100
\end{aligned}
$$

Number of weighted positive or negative emotionassociation votes per colour:

Total for all 4 positive emotions of (No. of votes for each positive emotion per colour * \% of importance of that emotion) -

Total for all 4 negative emotions of (No. of votes for each negative emotion per colour * \% of importance of that emotion)

\section{Discussion of results}

For the first part of this study, the results obtained and transcribed from the focus groups and interviews with under graduates, post graduates and adult learners were matched with those obtained from the questionnaire open-ended questions. The resulting propositions offered by students to enhance the interior and exterior design of learning spaces within 3D VLE university campuses, were divided into 124 design features that were consequently grouped into 11 major categories as follows:

\subsection{Recommended design categories and their features}

The proposed 124 design features suggested by students and staff to be used within educational buildings were divided into the following 11 major categories:

- The architectural style (e.g. modern, classic, gothic) and shape of the building (e.g. circular, square, use of columns etc.)

- Wall design, finishing and colours

- External environment elements of design

- Seating arrangements and shapes

- Window styles, shapes and lighting intensity

- Internal space design factors (e.g. size and dimensions)

- Roof and ceiling design, finishing and colours
- Floor design, finishing and colours

- Circulation design specifications (e.g. stairs, corridor width etc.)

- Internal design elements (e.g. availability of desks, screens, boards etc.)

- Entrance design (e.g. width, height, shape, doors, ease of accessibility etc.)

These categories represent all the design features of a 3D virtual educational building that are of interest to the student or teacher within a 3D VLE to provide satisfaction and contentment during an elearning session within that space.

It was made clear to all participants that the aim of this research was not to focus on specific features and therefore limit the scope of the research outcomes. The main objective was, after identifying design features that could be grouped on the previously defined categories, to map out the effects of such features on the learning experience of participants. Emphasis was also given on the investigation of how such innovative environments would contribute to the transformation of e-learning supportive technologies.

One emerging significant outcome was the extreme similarity in results between the post graduate student groups and the adult learner groups regarding their responses to the survey open-ended questions and suggestions during focus groups and interviews. Hence the following figures represent the average findings for all participants within the study.

\subsection{Number of votes for each design category}

Figure 1 below illustrates that the highest overall number of suggestions and preferences were directed towards enhancement in the architectural style (including shape of the building), and the interior wall design of the learning space. This is evident from the number of different features proposed in each of these categories in the questionnaires, and also by the high total number of votes offered especially by post graduates and adult learners. Apparently wall design (including colours and textures) is of higher significance to under graduates; maybe due to the fact that this is the immediate visual element they are in contact with during elearning sessions, while age maturity of the other two groups of students allow them to perceive the overall style and shape of the space as well. Favourable categories are also those related to the external environment and seating arrangements. It can be seen that the younger the age group of the students, the more important the external environment is to them, e.g. presence of greenery, water elements, landscaped design etc. This maybe due to the enjoyment of students from flying and exploring around in the 3D virtual environment, not just remaining focused inside the $3 \mathrm{D}$ learning space they are taking their session in. 


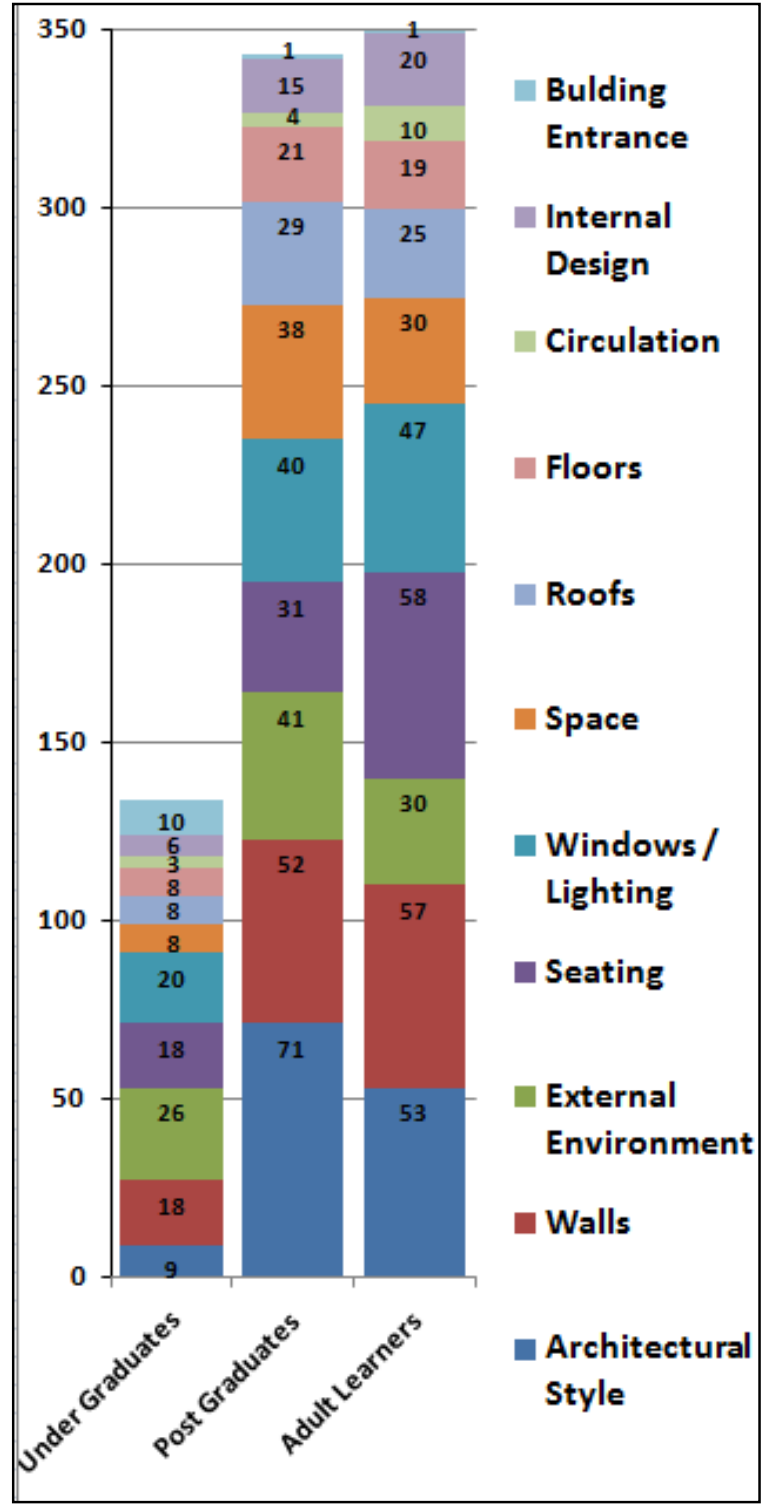

Figure 1. Number of votes provided for each architectural design category by each group of students

Furthermore, seating is the highest scoring category with adult learners, indicating practicality of this age group to require functional and comfortable seating arrangements during a virtual elearning session. Window design and effect of internal lighting also appears to be very important, for although not as many different number of design features were suggested for this category in the questionnaires, it gathered a high number of votes compared to overall votes, especially with under graduates. Again similar to the case with walls, this can be attributed to the fact that windows are in the immediate line of sight of students hence of high importance to them. The internal space dimensions, roof and floor design also collected votes from a considerable number of applicants. Their decreased significance may be due to roof and floor not being in direct line of vision of users, and dimensions are a background not immediate object of realization and perception for users. Least in value to post graduates and adult learners emerge the issues related to circulation and the building entrance design. This is expected since these users are more focused on design aspects of the learning space itself in which they are receiving their e-learning session. Whilst due to the exploratory nature of under graduates, building entrance appears significant to their desire to move or fly around in and out of the 3D virtual learning room. In general it can be seen that post graduate and adult learner results were very similar compared to under graduate results. Furthermore post graduates and adult learners appear to be more interested in the architectural design features of the space than the under graduates evidenced by the considerable difference in total number of votes provided by the two groupings of students mentioned.

One of the authors' concerns was the fact that the architectural experience of the learning space was, as expected, in the form of numerous interweaved features. Although in further pilot studies certain elements were highlighted and research was narrowed down to assess the effects of specific features, it was clear that users had a plethora of stimulating interface aspects drawing their attention.

\subsection{Highest three features in each design category}

Figure 2 illustrates the number of votes offered for the top 3 features recognized in each design category, suggested by the participating students. The features are ordered in descending order according to number of votes. As apparent from the results, the features with highest intensity of votes occur within the design categories explained earlier as the mostly preferred, thus coinciding with previous results in this paper. The features achieving highest preference were those related to the architectural style, wall design, seating, windows and lighting, and the external environmental design.

The highest inclination was towards having strong internal lighting within the e-learning space. When asked in person, students stated that this helped them to concentrate, especially if the lighting could be emulated to appear as natural not synthetic. There also seems to be an apparent fondness for plain modern and classical building styles. When asked in focus groups and interviews to elaborate on this issue, students and staff commented that imaginative and untraditional style buildings make them uncomfortable to be in and cause distraction and uneasiness during e-learning sessions. Additionally, the usage of brighter wall colours appeared to be high on the preference list. Further 
remarks added that brighter colours for ceilings and floors were also favoured providing liveliness unlike dull interiors or dark finishing which makes the environment "gloomy and put us to sleep".

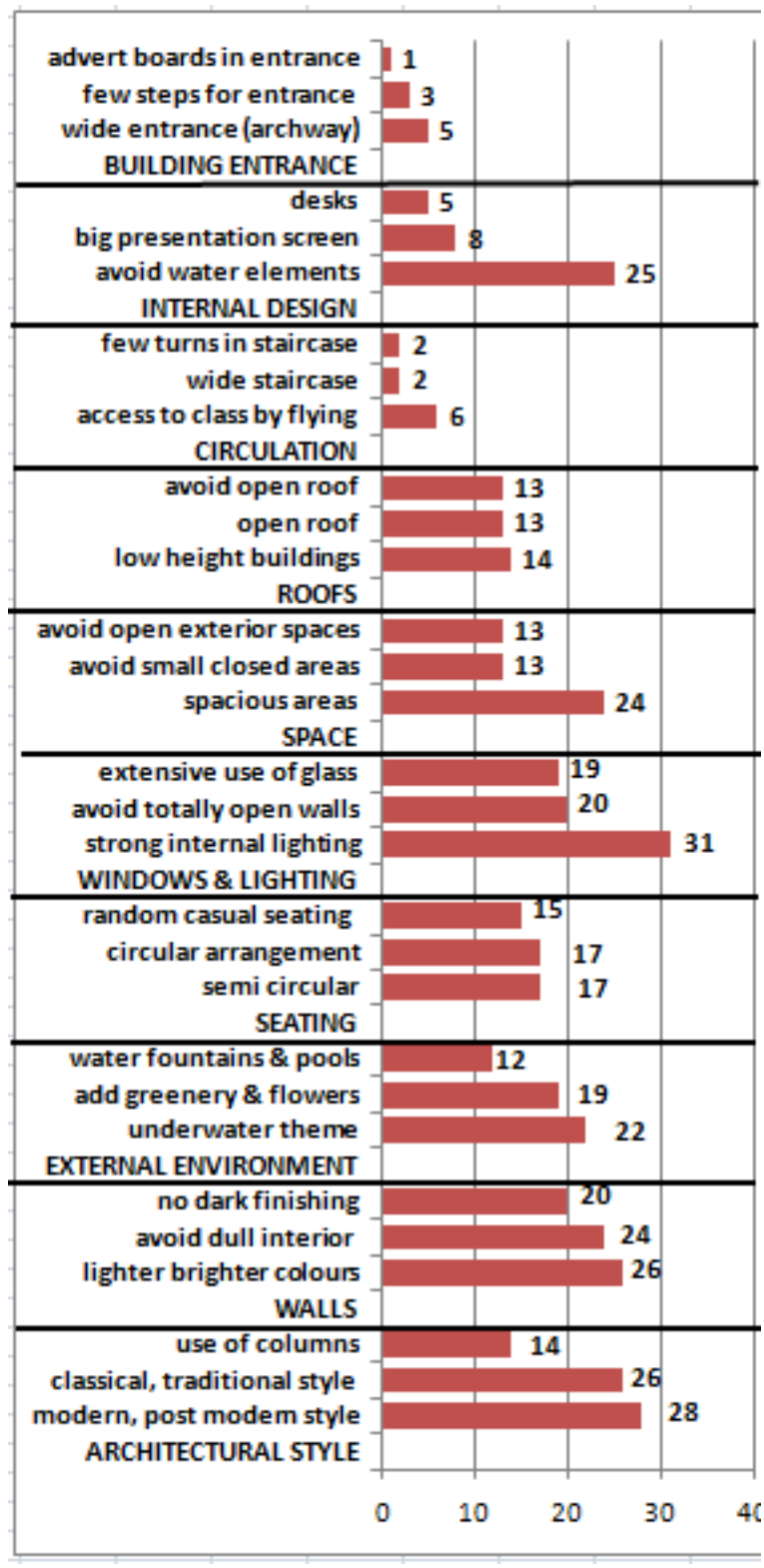

\section{Figure 2. Percentage votes of the top 3 features in each architectural design category}

A surprisingly high number of votes were given to avoiding presence of water elements such as fountains etc within the interior of an educational space. Explanations for this included that this creates a distraction for students. On the other hand, total submergence of the building underwater, or even just presence of surrounding pools, water fountains or even the sea in the surroundings was highly commended as very inspirational and cheerful.
Presence of environmental greenery and flowers was also recognized as a joyful must.

On a separate note, spacious areas with extensive use of glass for walls were highly praised, adding to the feeling of comfort during an e-learning session. However, presence of completely open walls was not a favourite due to sensations of insecurity and instability that accompany it, in students and staff opinions. Along the same vein, there was equally divided opinion amongst participants whether to use completely open roofs or not. Some said it added to the feeling of insecurity causing distraction, whilst others commented that it provided spaciousness and peacefulness due to blending with the sky. Low height buildings were also preferred.

As for seating arrangements, most users preferred circular and semi-circular arrangements, but definitely not linear ones. Random seating was also suggested for more informal sessions of e-learning that involve discussions etc.

The mostly recommended features related to circulation within 3D virtual e-learning buildings were ease of access to class by flying rather than by stairs, elevators or corridors. This would hence necessitate, as mentioned earlier, extensive use of glass windows or open wall areas. In case of use of stairs, wider shorter and fewer turns, flights and corridors were preferred. Building entrances should also be wide with few steps for entry. Students explained that narrow corridors, doors and flights of stairs were very inconvenient for their avatar movements and manipulation within the 3D space.

There were also some minor requirements for other elements which are not directly related to design of the space, such as advertisement and bulletin boards for student orientation.

There are several reservations regarding the different genres of 3D VLE and virtual world users which were witnessed during the previous data collection phase discussed in this paper. It is imperative for designers of such spaces to keep in mind that (i) not all users of a certain generation can be familiar with gaming features and interfaces, (ii) as with social networking the penetration of serious gaming and virtual worlds varies significantly in different regions, (iii) the expectation of technologically savvy users in certain disciplines does not apply in such demanding applications, (iv) the ability to transform traditional learning activities from 2D to 3D VLEs and (v) the skills required to engage in synchronous learning activities while using a 3D VLE interface.

\subsection{Number of votes for each positive and negative emotion per colour}




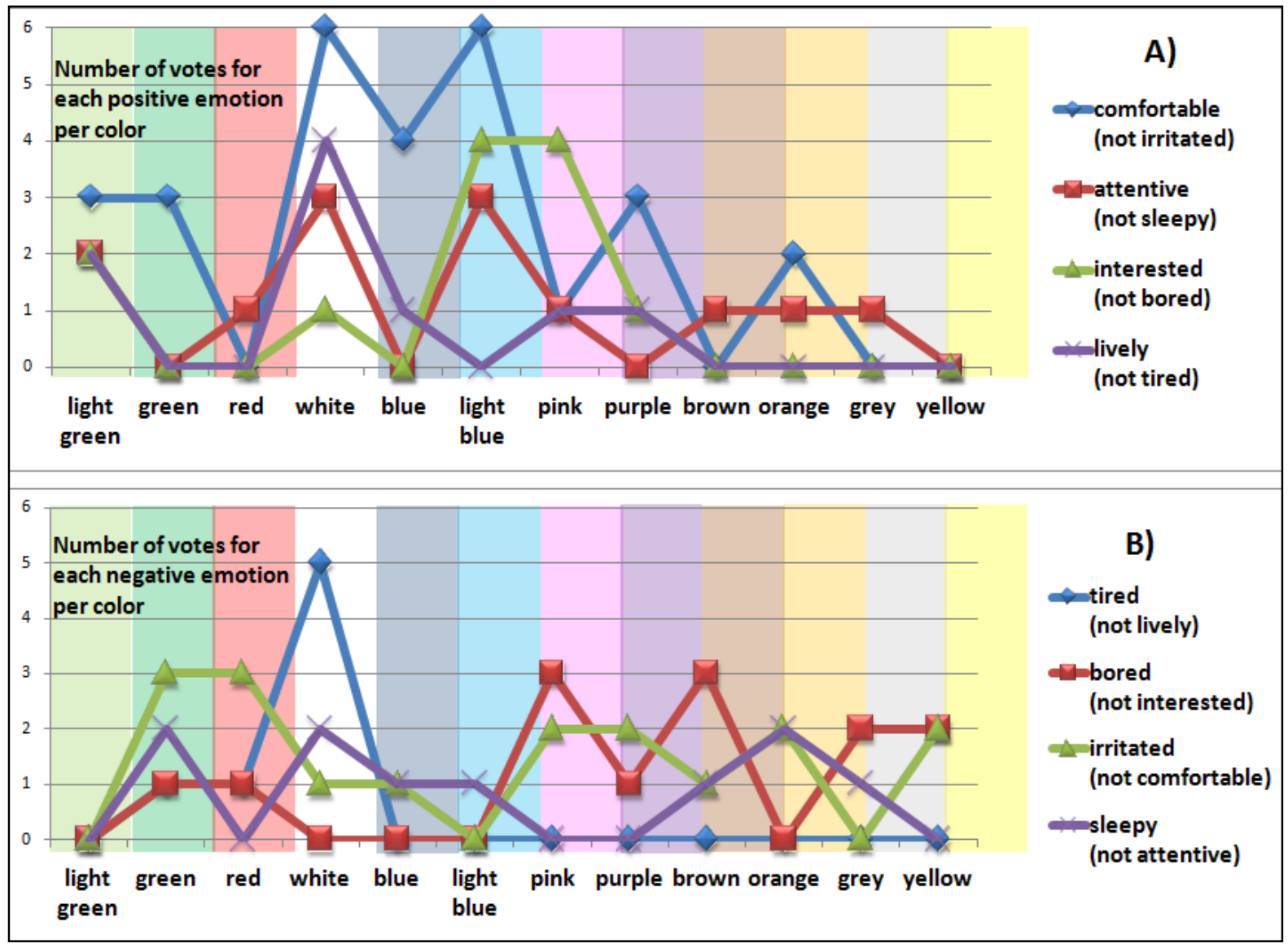

Figure 3. A) Number of votes provided by overall students for each positive emotion per colour B) Number of votes provided by overall students for each negative emotion per colour

Regarding the second part of the study demonstrated in this paper, which concerns students' negative and positive emotion associations with colour of the 3D learning space walls, floor and ceiling (as an example of an architectural design feature), the following results were derived. The significance of these results lies in the correlation of positive and negative colour associations respectively with affordances and constraints for elearning inside 3D VLEs.

Figure3 illustrates number of votes for each positive and negative emotion that students experienced during their e-learning sessions using each of the 12 tested colours mentioned formerly. A higher number of positive emotion-associated votes is an indicator for greater satisfaction of students from the colour of their $3 \mathrm{D}$ virtual learning space. Figure 3A confirms that white and light-blue evoke highest number of positive emotions, specifically comfort followed by attentiveness. However, lightblue seems to induce less boredom (more interest) than white - maybe due to the latter being a neutral colour - while white gives a higher feeling of liveliness. These results are consistent with "reallife" findings elaborated earlier, which also coincide with the considerable comfort experienced with green hues virtually and physically. Additionally pink provided more attentiveness than most other colours in the virtual learning space analogous to providing creativity in the physical world as shown from literature. However surprisingly, purple and orange were more favoured here than "real-life" inducing comfort. Expectedly, brown and grey were least preferred, whereas red was less likeable virtually than in "real-life". Physically, yellow, as an individual colour, was found to encourage creativity while green-yellow was considered repulsive. However virtually, results show that yellow and dark-green in general are not recalled by students to induce any positive emotions during e-learning.

Figure3B triangulates the preceding findings denoting least negative emotions experienced with light-blue and light-green, hence emphasizing their favourability. Students appear to have lower inclination towards most other virtual digital colours characterized by presence of some negative emotions e.g. boredom with brown, irritability with dark-green and red which corresponds with findings from Figure3A. Participants were differently opinionated towards pink which provided almost equal amounts of opposing boredom and interest emotions. Strikingly unexpected results were obtained for 


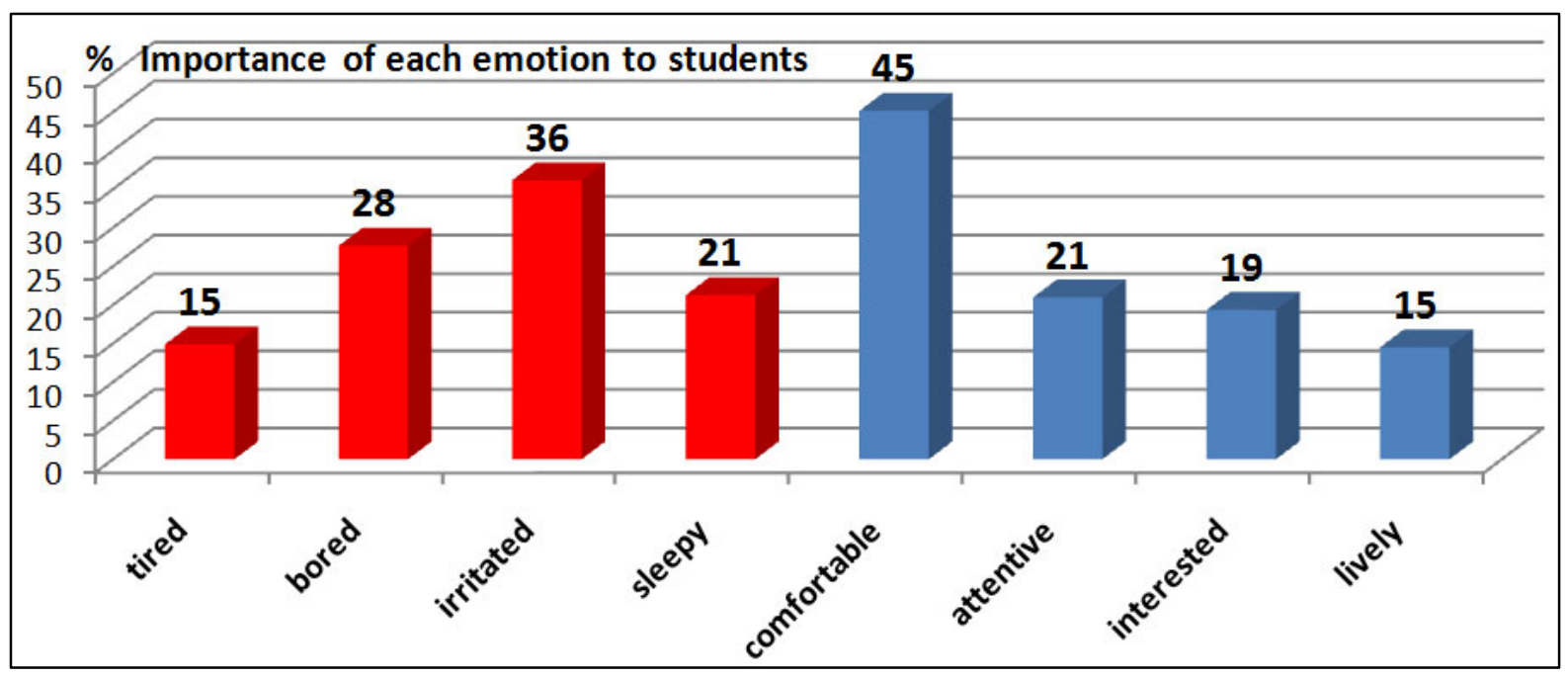

Figure 4. Percentage of importance of each colour-associated emotion as calculated from students' votes

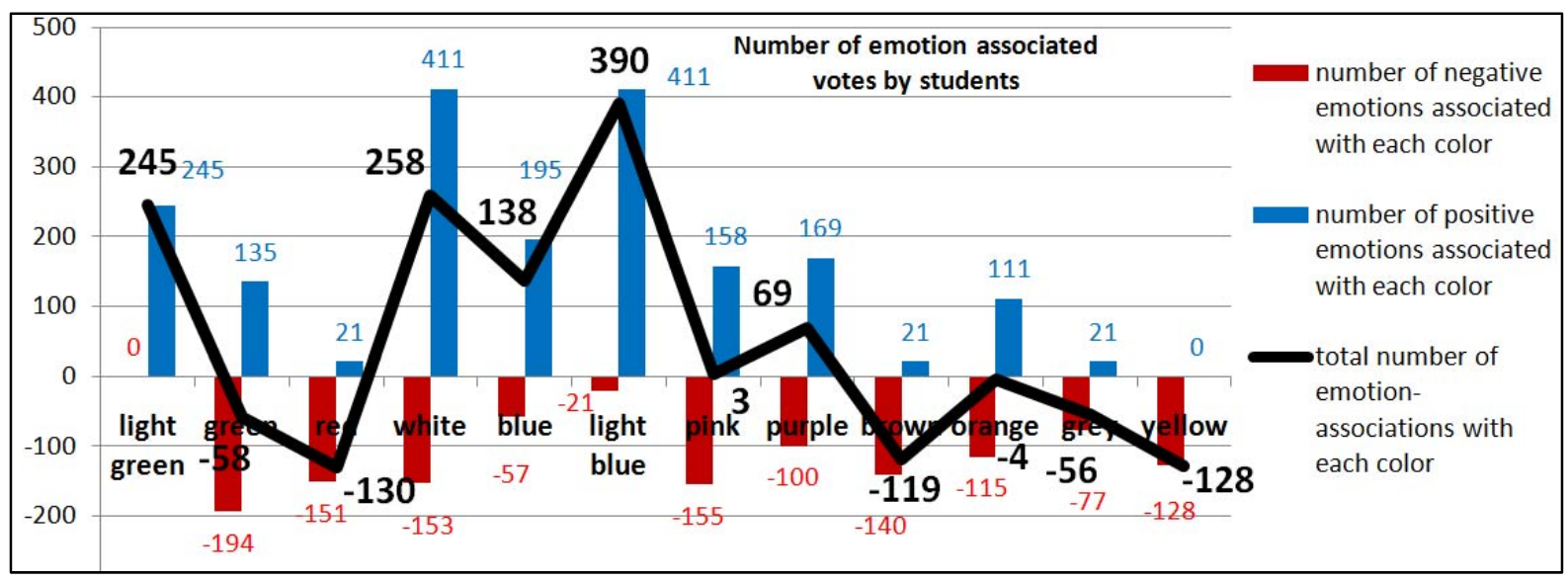

Figure 5. Number of weighted positive and negative emotion-association votes, and their difference provided by all students for each colour

white which induced highest level of feelings of tiredness. However these findings are not inconsistent with results from Figure3A and physical world literature which all denote high student satisfaction from white, as will be explained consequently.

4.5. Number of weighted positive and negative emotion-association votes based on percentage of importance of each emotion

A crucial factor in translating the impact of the colour-associated emotions on overall satisfaction of students from a particular learning space colour, is the relative percentage importance of each positive or negative emotion to the participants, calculated by equation(1). Figure4 demonstrates that comfort is the most important positive emotion required by students to provide e-learning affordances and capabilities, whilst irritation followed by boredom is the most undesirable negative emotion that inhibits students' contentment from the learning space and thus can act as a constraint or limitation to the e-learning experience.

This can elucidate, as follows, how white remains an overall highly recommended colour despite the negative emotions experienced within its presence. Figure5 displays total number of positive and negative votes/colour, multiplied by a certain weight factor representing percentage importance of each emotion (as calculated by equation 2). This shows the actual impact of these emotions experienced by students per colour. As a result, it is evident that since the negative tiredness sensation recorded with white has a relatively low percentage of importance compared to the high percentage of importance displayed for comfort (highly recorded with white); "tiredness" only slightly affects the overall number of emotion-association votes for white, calculated 


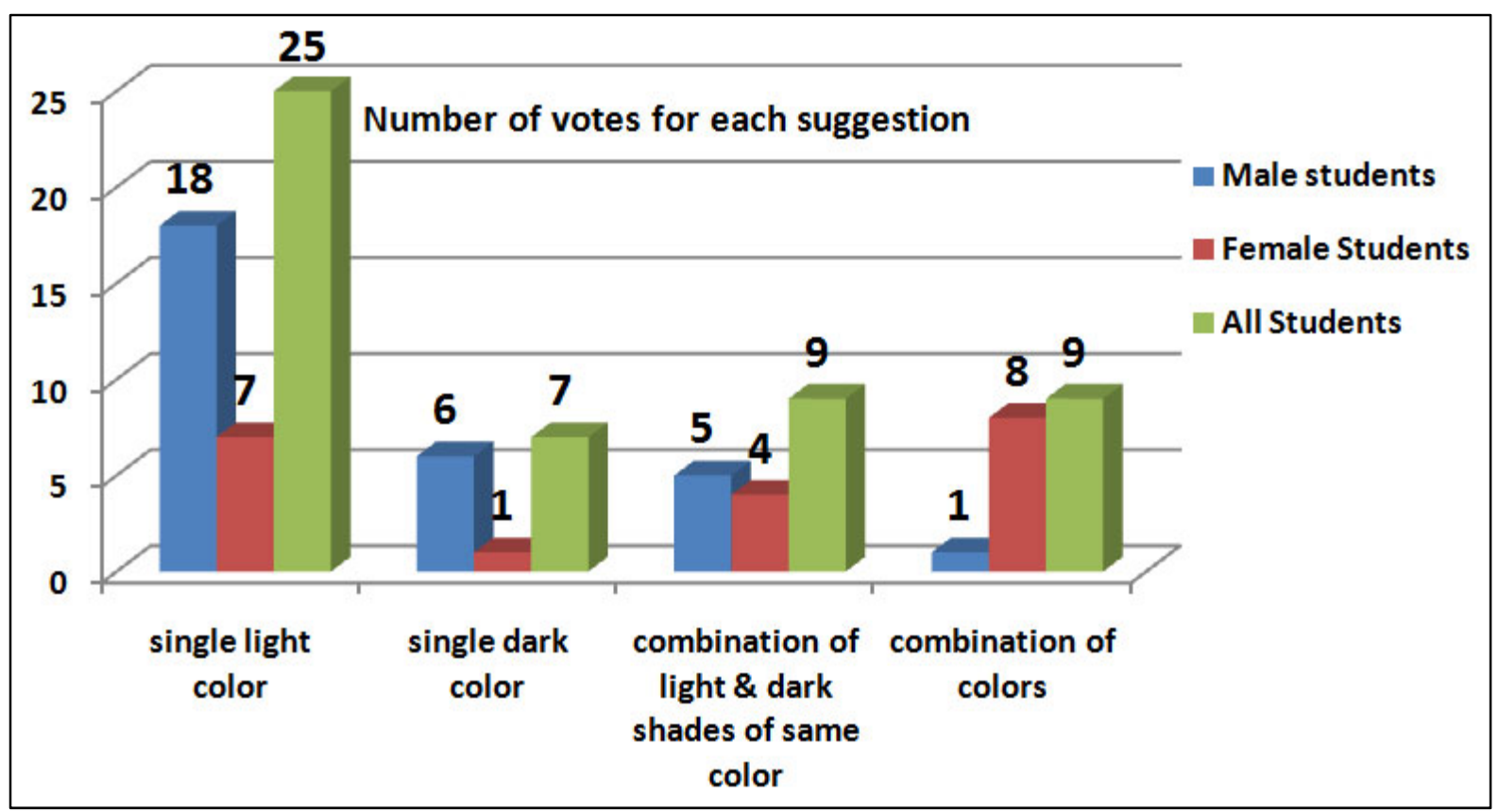

Figure 6. Student Propositions for colour design of 3D virtual Learning Spaces

from the difference between the positive and negative. This rendered white still highly desirable following light-blue, as apparent from figure5. Lightgreen and blue are next in popularity, while the rest of the colours either provide no overall positive or some negative sensations.

\section{Conclusion}

As evident from the first half of the study elucidated in this paper, architectural style, wall design, window design and lighting, and seating arrangements appear to have piqued the most interest of many participants for architectural design enhancement of 3D e-learning spaces within 3D VLEs. This may be due to the fact that these factors are the closest in proximity and perspective to the eye during presence within the virtual campus. This hypothesis can be further validated by the fact that students generally indicated that bright lighting and colours generate a feeling of comfort and joy during an e-learning session. Also spacious, great height shapes contributed to concentration and elimination of distraction. Thus, the top design features, specifically preferred by students, include predominantly the presence of strong internal lighting, semi circular rows for seating, using a simplistic modern or classical architectural space design rather than imaginative or untraditional styles, and using lighter brighter colours for the internal walls, ceiling and floor finishes.

The authors have identified opportunities for further research in terms of clustering pilot study participation to reflect the different needs of varying user groups. Further work is underway to establish how 3D VLEs (i) can support students of different disciplines, (ii) are perceived by different types of academic and academic related staff and (iii) designers of 3D VLEs attempt to address learning needs in such environments.

In conclusion, based on evidence provided by this paper showing eagerness of participants to suggest propositions for enhancement of 3D educational buildings design, it can be inferred that the internal and external architectural design characteristics of a 3D educational facility erected within a 3D VLE have an impact on the satisfaction and contentment of users of this e-learning space, namely students and members of faculty. Hence it is imperative to further investigate effect of different architectural design elements on e-learning experiences of students, to be able to issue recommendations for enhancement of the digital design of this genre of $3 \mathrm{D}$ buildings for the better benefit of its users.

The second part of this study aimed at investigating an example of an architectural design element in more detail, hence the choice to define colours which invoked best and worst sensations for students during e-learning sessions in 3D virtual learning spaces within 3D VLEs e.g. Second Life. Pinpointing these colours would help identify best practices for architectural digital colour design of these $3 \mathrm{D}$ virtual spaces to provide affordances and enhancements for the students' learning experience within them. Findings demonstrated that light-blue conjured highest positive emotions of comfort, attentiveness and interest which represent highest importance for students; (liveliness, which is of low 
importance, and negative emotions were non-existent for this colour). This was followed by white, light green, blue and controversially pink. Colours invoking most negative sensations were yellow, red and brown. All these preceding results are analogous to those researched in "real-life", with the exception of purple and orange which are more desirable virtually. Results also show that there is a general preference for using single light colours.

Future research includes studying colourassociated emotions and satisfaction of students from combinations of colours and different hues of the same colour. Moreover, variances of results with students' age, gender and field of study can be further explored. Effects of other architectural elements of 3D virtual learning spaces on students' e-learning are also currently being investigated, e.g. shape, dimension ratios, textures, lighting, percentage open spaces, seating arrangements etc., to derive best design guidelines for erection of $3 \mathrm{D}$ virtual educational facilities that would boost student satisfaction and learning experience.

\section{References}

[1] N. Saleeb, G. Dafoulas, "Pedagogical Immigration to 3D Virtual Worlds: a Critical Review of Underlying Themes and their Concepts", Proceedings of IEEE International Conference on Information Society (i-Society 2010), London, UK, 2010.

[2] A. H. Bridges, D. Charitos, "The Impact of form on Movement within Virtual Environments, Automation in Construction", Elsevier Science BV, vol. 10, no. 5, Amsterdam, 2001.

[3] J. P. Eberhard, “A Place to Learn: How Architecture Affects Hearing and Learning”, The ASHA Leader, vol.13, no. 14, 2008, pp.26-27, 29.

[4] N. Saleeb, G. Dafoulas, "Relationship between Students Overall Satisfaction from 3D Virtual Learning Spaces and their Individual Design Components", International Journal of Computer Science Issues (IJCSI), vol. 7, no. 4, 2010.

[5] G. Minah, "Colour as Idea: The Conceptual Basis for Using Colour in Architecture and Urban Design”. Journal of Color: Design and Creativity, issue 2, 2008.

[6] S. Minocha, N. Mount, "Design of Learning Spaces in 3D Multi-user Virtual Environments". JISC Learning and Teaching Committee; The e-Learning Programme, 2009.

[7] R. Reffat, "Collaborative digital architecture design teaching within virtual environments", CAADRIA 2005, Computer Aided Architectural Research in Asia, India, 2005, pp. 65-74.

[8] R. Reffat, Y. Khaeruzzaman, E. El-Sebakhy, P. Raharja, “Augmentation of Real-Time 3D Virtual Environments for Architectural Design at the Conceptual Stage”, Journal of Information Technology in Construction
-ITcon, Vol. 13: pp.553-563, Special Issue Virtual and Augmented Reality in Design and Construction, 2008.

[9] "Second Life Buildings and Real Estate Market 1st Quarter, Market truths Limited”, State of the Art Market Research and Analysis, 2009.

[10] M.D. Dickey, "An architectural perspective for the design of educational virtual environments". Journal of Visual Literacy. vol. 24, issue 1, 2004

[11] A.H. Bridges, \& D. Charitos, "On Architectural Design of Virtual Environments”, Design Studies, Elsevier Science Ltd., vol. 18, issue 2, 1997, pp.143-154

[12] S. Minocha, A.J. Reeves, "Interaction design and usability of learning spaces in 3D multi-user virtual worlds”, Human Work Interaction Design, Pune, India, 2009.

[13] A. G. Walstrom, "Color in the Classroom: How schools are using color to create ready-to-learn environments". Retrieved 10 January, 2011, from http://www.swstir.com/color-smarts/article/color-in-theclassroom/, 2009.

[14] N. Kaya, "Relationship between color and emotion: a study of college students", College Student Journal. Retrieved 5 January, 2011, from http://findarticles.com/p/articles/mi_m0FCR/is_3_38/ai_n6 249223/, 2004.

[15] W. L. Wilder, "Importance of Color in Web Design". Retrieved $15 \quad$ January, 2011 from http://www.cyberindian.com/web-designing/importanceof-color-in-web-design.php, 2008.

[16] C. J. Boyatzis, R. Varghese, “Children's emotional associations with colors ", The Journal of Genetic Psychology, vol. 155, no. 1, 1994, pp.77-85.

[17] A. Failey, D. E. Bursor, R. A. Musemeche, "The impact of color and lighting in schools". Council of Educational Facility Planners Journal, 1979, pp. 16-18.

[18] F. Birren, "Color Psychology and Color Therapy". New Hyde Park, New York: University Books, Inc., 1961.

[19] M. Terwogt, J. Hoeksma, "Colors and emotions: preferences and combinations". Journal of General Psychology, vol. 122, 1995, pp. 5-17.

[20] D. K. Ballast, "Interior design reference manual”. Professional Pub. Inc.: Belmont, CA., 2002. 